\title{
Actitud hacia las TIC y hacia su integración didáctica en la formación inicial docente
}

Attitude towards ICT and toward its didactic integration in the initial teacher training

\section{Volumen 18, Número 3 \\ Setiembre-Diciembre}

pp. 1-29

\section{Este número se publica el 1 de setiembre de 2018 \\ DOI: https://doi.org/10.15517/aie.v18i3.34437}

Hugo Gonzalo Tapia Silva

Revista indizada en REDALYC, SCIELO

Revista distribuida en las bases de datos:

LATINDEX, DOAJ, REDIB, IRESIE, CLASE, DIALNET, SHERPA/ROMEO, QUALIS-CAPES, MIAR

Revista registrada en los directorios:

ULRICH'S, $\underline{\text { REDIE}}, \underline{\text { RINACE}}, \underline{\text { OEI, MAESTROTECA, PREAL, }}$ 


\section{Actitud hacia las TIC y hacia su integración didáctica en la formación inicial docente}

Attitude towards ICT and toward its didactic integration in the initial teacher training

\section{Hugo Gonzalo Tapia Silva}

Resumen: El ensayo aborda un marco teórico para el desarrollo de los constructos actitud hacia las TIC y actitud hacia la integración didáctica de las TIC en la formación inicial docente. Para ello, se presenta la importancia de desarrollar una actitud positiva hacia las TIC - A(TIC)+-y una actitud positiva hacia la integración didáctica de las TIC - A(IntDid)+ - . Establecidos los constructos, expresamos la necesidad de considerar en la formación docente variables y orientaciones que influyan en el logro de una actitud positiva hacia las TIC en educación, tales como conocimientos y habilidades TIC, la disciplina formativa o las creencias sobre el aprendizaje. Posteriormente, se proponen acciones para desarrollar la actitud positiva hacia las TIC durante la formación inicial docente. Se concluye la necesidad de diseñar el proceso formativo de futuros profesores, a partir del desarrollo de conocimientos y habilidades TIC, en la formación pedagógica, disciplinar y tecnológica desde una perspectiva sobre cómo aprender utilizando tecnologías.

Palabras clave: tecnología educativa, actitud docente, formación inicial docente, didáctica

Abstract: This essay presents a theoretical framework to develop the constructs: attitude towards ICT and attitude towards the didactic integration of ICT in teachers' education. We discuss the development of a positive attitude towards ICT $(+A(I C T))$ and a positive attitude towards the didactics integration of ICT $(+A(I n t D i d))$. Further, we claim that a positive attitude towards ICT is influenced by variables and orientations such as knowledge and ICT skills, subject knowledge, or beliefs about learning. In addition, we propose some actions to develop a positive attitude towards ICT in teachers' education programs. We conclude that the design of teachers' education curriculum should include the development of ICT knowledge and skills closely connected to pedagogical knowledge, content knowledge, and technology as well as the provision of an approach regarding how to use technology to assist learning.

Keywords: educational technology, teacher attitude, initial teacher education, didactic

\footnotetext{
1 Investigador y académico de la Universidad de La Serena, en el Instituto de Investigación Mutidisciplinar en Ciencia y Tecnología, Chile. Doctor en Educación por la Universidad de Navarra, España.
}

Dirección electrónica: hgtapia@userena.cl

Ensayo recibido: 11 de abril, 2018

Enviado a corrección: 11 de junio, 2018

Aprobado: 27 de agosto, 2018 


\section{Introducción}

Actualmente se evidencia una importante presencia de las tecnologías de la información y comunicación (en adelante TIC) en diferentes instancias de la sociedad, incluyendo los ámbitos educativos (Hinostroza, 2017; Unesco, 2014). Es en estos ámbitos educativos que la formación inicial de profesores se convierte en un aspecto crítico, que debe considerar las características que los usos de las TIC han ido estructurando: acceso masificado a Internet, presencia de tecnologías en las escuelas, aumento en la frecuencia de uso de tecnologías, y tipos de uso emergentes. Tales cuestiones deben, con el fin de mejorar la educación escolar, incorporarse en el diseño de la formación del profesorado para su posterior integración en prácticas educativas y pedagógicas (Amelii, Reyes y Ríos, 2017; Rozo y Prada, 2012). Si bien se reconoce su importancia educativa, todavía se observa un estado inicial en este proceso (Brun, 2011), por lo que es necesario identificar cuáles son las variables que pueden potenciar su integración los diseños de aprendizajes y su implementación en el aula. Al respecto, dificultades tales como el tiempo y dedicación del profesorado, competencias TIC, recursos disponibles o la coordinación académica, entre otras, han sido identificadas (González Pérez, y De Pablos Pons, 2015; Raman y Yamat, 2014).

En el caso de la formación inicial de profesores, se han llevado a cabo trabajos que, por una parte, identifican los conocimientos y habilidades TIC que se consideran necesarios para la profesión docente (Unesco, 2011), y, por otra, expresan la preocupación por las estrategias adecuadas para la implementación de procesos formativos que permitan lograrlos (Rizza, 2011). Existe, asociado a estos conocimientos y habilidades, un constructo que podría orientar y aumentar la comprensión del uso de las TIC en los procesos educativos y, por tanto, en la formación de profesores. Nos referimos al concepto de actitud (Albirini, 2006; Drossel, Eickelmann, y Gerick, 2017; Guzman y Nussbaum, 2009).

¿Qué es la actitud y cuál sería su relación con las TIC? ¿Cuál sería el aporte del constructo actitud a la formación de profesores para el uso de las TIC? ¿Qué variables influirían en su construcción de la actitud? ¿Qué estrategias pueden realizarse para orientar la formación de los profesores para lograr una actitud que favorezca la integración didáctica de las TIC en los procesos de enseñanza-aprendizaje? El presente ensayo busca responder a estas preguntas con el fin de mejorar la formación del profesorado, siendo parte de una investigación que busca identificar el rol de la actitud en el proceso de integración de las TIC durante la formación docente con miras a una futura práctica pedagógica. 


\section{Desarrollo del tema}

\subsection{Actitud hacia las TIC}

Una línea actual de investigación sobre la influencia de las TIC en la sociedad aborda las características de las nuevas generaciones de niños y jóvenes en torno al uso de las TIC en sus procesos de aprendizaje. Si bien hasta hace algunos años había escasas investigaciones sobre las características de los profesores en formación que pertenecen a estas nuevas generaciones (Lei, 2009), en los últimos años ha emergido el concepto de Millennials teachers para referirse a quienes nacieron a partir del año 1990 y se están formando o ya ejercen el profesorado (Clark y Byrnes, 2015; Johnson, 2006).

Aun cuando quienes pertenecen a las nuevas generaciones no posean habilidades superiores en el uso de las TIC (Calvani, Fini, Ranieri, y Picci, 2012; Margaryan, Littlejohn y Vojt, 2011), no se puede negar que su relación con las tecnologías difiere de la que poseen quienes nacieron hace más de tres décadas. En este contexto, cabe preguntarse por su actitud hacia las TIC, pues esta se encontraría relacionada con el uso que realizarán en su formación y su futuro rol docente (Abbitt, 2011; Albirini, 2006; Avidov-Ungar y Eshet-Alkakay, 2011; Drossel, et al., 2017; Dunn y Rakes, 2011; Enochsson y Rizza, 2009; Knezek y Christensen, 2008; Teo, 2008; Webb y Cox, 2004).

\subsubsection{Concepto de Actitud}

Tal como otros conceptos abstractos - conocimiento y aprendizaje, por ejemplo - el concepto actitud es equívoco debido a su complejidad (Guitart Aced, 2002; Henerson, Morris, y Fitz-Gibbon, 1987). Existen, al menos, tres perspectivas principales sobre la estructura de las actitudes: a) una entidad con tres componentes, b) tres entidades separadas y c) la variable latente (Cheung, 2009).

La primera perspectiva considera que la actitud es una única entidad que está constituida por tres componentes: el afecto -el sentir frente a...-, la cognición -un pensamiento o creencia acerca de...- y la conducta -un actuar ante...- (Borkowski, 2005). Este modelo de comprensión de la actitud fue popular en la década de 1960.

Una segunda perspectiva considera estos componentes (afecto, cognición y creencia) como entidades distintas, en la que la actitud se reserva para el componente "afecto", y se entiende como la cantidad de sentimiento a favor o en contra de un objeto u objetivo afectivo (Henerson et al., 1987). Como ha observado Cheung (2009), para algunos investigadores esta perspectiva no recoge la complejidad que una actitud posee. 
Para la tercera perspectiva, la actitud sería un resumen de la información observable tras las respuestas cognitivas, conductuales y afectivas que estimulan ciertos objetos o eventos, las que no se producen siempre en conjunto, si bien se encuentran mutuamente asociadas (Cheung, 2009; Guitart Aced, 2002; Haddock y Huskinson, 2004; Maio y Haddock, 2004). La información afectiva hace referencia a los sentimientos o actitudes - ansiedad, alegría, miedo, entre otros -; la información cognitiva a las creencias asociadas a un objeto, persona o acto; la información conductual a las conductas pasadas relativas al objeto al que se dirige la actitud. Estos tres componentes se encuentran correlacionados, de forma que un sentimiento positivo no podría implicar conductas o creencias negativas (Haddock y Huskinson, 2004; Maio y Haddock, 2004).

En línea con esta tercera perspectiva, para Knezek y Christensen (2008), la actitud es una predisposición (o tendencia) que puede ser favorable o en contra. Esta predisposición puede estar dirigida a) hacia un objeto en concreto (situación, idea, sujeto, entre otros) como consecuencia de su evaluación (Guitart Aced, 2002; Teo, 2008), lo que denominaríamos $A(0)$; o, por otra parte, b) hacia el desarrollo de una conducta, lo que denominaríamos $A(c)$ (Kroenung y Bernius, 2012). Esta predisposición surgiría, por tanto, como resultado de los afectos, los conocimientos y las conductas vividas por el sujeto. En la siguiente figura 1 esquematizamos la actitud como resultado de tres tipos de condiciones.

Figura 1

Componentes de la actitud

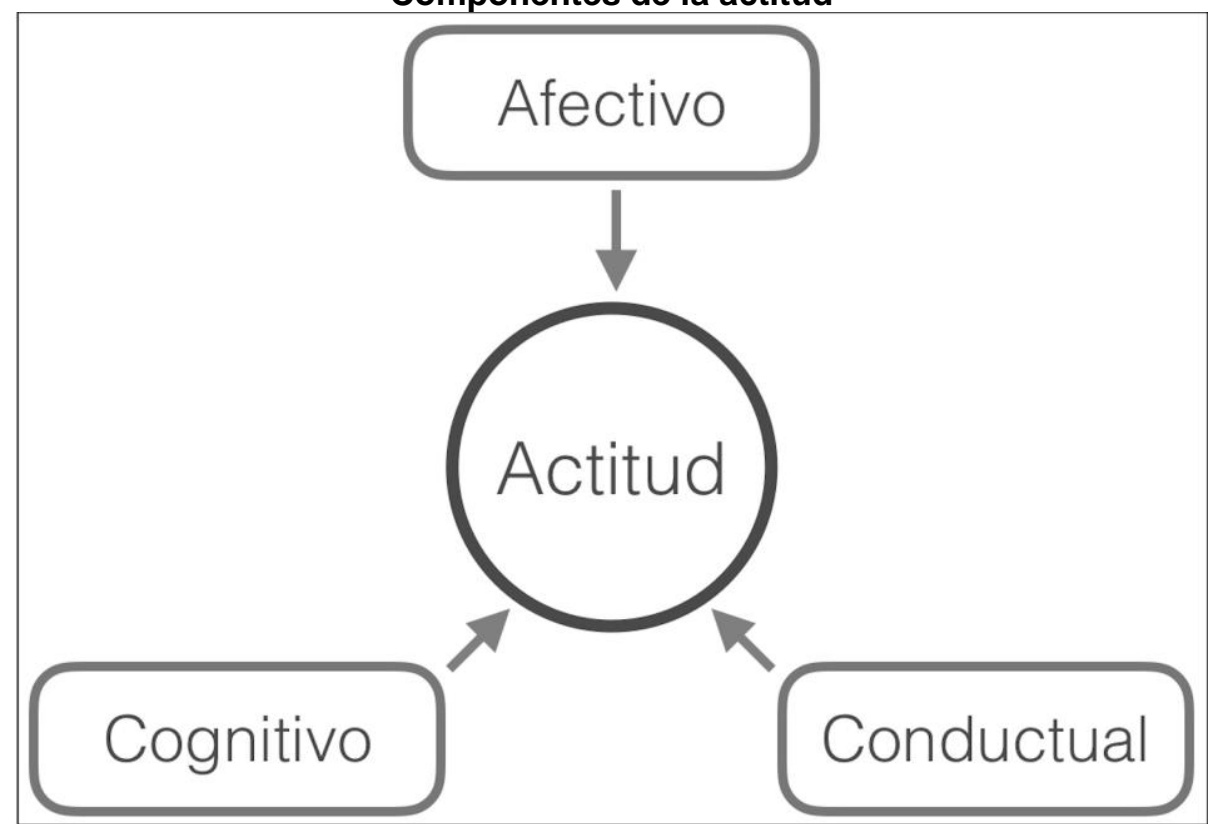

Fuente: Elaboración propia a partir de los modelos propuestos (2018). 
En general, se reconoce que el sistema de actitudes dotaría el comportamiento y orientación del individuo de coherencia e integridad. Así, se ha comprobado que las actitudes sirven para ordenar y dar significado a aspectos del medio social en el que la persona se mueve, lo que permite dirigir su percepción sobre aquello con lo que se relaciona. Eso implica que podemos escoger estímulos del entorno en función de las actitudes que tenemos (hacemos una selección de los mismos), o podemos interpretar algo que nos rodea en una dirección determinada a partir de las mismas (Guitart Aced, 2002).

A partir de las teorías expuestas, consideramos que una actitud es una respuesta 0 tendencia sistémica que se origina en un sujeto frente a un fenómeno u objeto. Las actitudes se caracterizarían por:

- ser aprendidas o adquiridas por el individuo a lo largo de su vida;

- pueden referirse a un objeto, $\mathrm{A}(0)$, o hacia el desarrollo de una conducta, $\mathrm{A}(\mathrm{c})$;

- implican respuestas a favor, $A(x)_{+}$, o en contra , $A(x)$-, del objeto o conducta;

- son estables, sistémicas y condicionarían otros procesos psicológicos.

\subsubsection{Actitud hacia las TIC y su Integración didáctica}

El concepto de Tecnologías de la información y la comunicación se entiende en variados sentidos, ya sea haciendo referencia al hardware o dispositivos que se utilizan, al software o aplicaciones que con que se generan contenidos, a los usos que se les dan, entre otros (Castro, Guzmán, y Casado, 2007). Para nuestra investigación, entenderemos como TIC a todo producto de la técnica que favorezca la creación, resguardo y/o consumo de recursos electrónicos o digitales.

A partir de lo anterior, podemos definir la actitud hacia las TIC en educación como una disposición que genera una respuesta. Esta respuesta puede ser a favor, la que denominamos Actitud positiva, o en contra, que denominamos Actitud negativa. Ambas actitudes pueden hacer referencia hacia las TIC en general, en adelante $A(T I C)$, o hacia su integración didáctica en los procesos de aprendizaje, en adelante $A$ (IntDid). Siguiendo la nomenclatura empleada, podemos esquematizar en la Tabla 1 como: 
Tabla 1.

Nomenclatura de Actitud hacia las TIC y Hacia la integración didáctica de las TIC Tipo de actitud Hacia las TIC Hacia la integración didáctica de las TIC

\begin{tabular}{lll}
\hline Actitud favorable (positiva) & $\mathrm{A}(\mathrm{TIC})+$ & $\mathrm{A}(\operatorname{IntDid})+$ \\
Actitud desfavorable (negativa) & $\mathrm{A}(\mathrm{TIC})-$ & $\mathrm{A}(\mathrm{IntDid})-$
\end{tabular}

Fuente: elaboración propia del autor (2018)

¿Cuáles pueden ser los factores que influirían en la actitud de los profesores hacia las TIC y hacia su integración didáctica? Entre los factores que inciden- influyen en una respuesta positiva o negativa- en los futuros profesores se encontrarían: los conocimientos y habilidades TIC que se poseen; la disciplina formativa; y sus creencias sobre el proceso de aprendizaje (Agyei y Voogt, 2011; Albirini, 2006; Hermans, Tondeur, van Braak, y Valcke, 2008; Jimoyiannis y Komis, 2007; Kim, Jung, y Lee, 2008; Sang, Valcke, van Braak y Tondeur, 2010).

Considerando que el éxito de un programa de formación depende, entre otros aspectos, de las actitudes de quienes se encuentran involucrados en él (Teo, 2008), es conveniente centrarse en los elementos que configuran $A(T I C)$ y $A(I n t D i d)$ para integrarlas en su formación y favorecer la construcción de una actitud favorable hacia ellas. En los apartados siguientes nos centraremos, por lo tanto, en los factores anteriormente mencionados, presentando propuestas para su consideración en la formación docente.

\subsection{Factores que influyen en la Actitud hacia las TIC}

\subsubsection{Conocimientos y habilidades TIC de profesores en formación}

Diversas organizaciones han seleccionado conocimientos o habilidades TIC que se deberían incorporar a la formación docente como son el International Society for Tecnology in Education de Estados Unidos (ISTE, 2008); el Ministère de l'Éducation nationale, de l'Enseignement supérieur et de la Recherche de Francia (MENESR, 2015); el Ministerio de Educación de Chile a través de su Centro de Educación y Tecnologías, Enlaces (MINEDUC y ENLACES, 2011) y la Unesco (2011). Tales conocimientos y actitudes van configurando un tipo de uso de las TIC en educación.

Al referirnos a los conocimientos y habilidades TIC de un profesor en formación hablamos tanto de los adquiridos previos a su ingreso a la formación inicial docente como de los logrados durante tal proceso formativo. En el caso de los segundos, se busca una adopción con perspectiva didáctica de las TIC enfocada a la enseñanza y el aprendizaje. A 
esto apuntan, en términos generales, diferentes propuestas de adopción de las TIC en la formación inicial de profesores (FIP) (Jung, 2005; Unesco, 2014)

Actualmente, quienes ingresan a la FIP han desarrollado un uso personal de las TIC, enmarcado en un contexto de cultura digital (Escofet Roig, García Gonzalez y Gros Salvat, 2011). En el ámbito educativo, no obstante, no se produce necesariamente un uso de las TIC para aprender, sino más bien de ámbito lúdico (Calvani et al., 2012; Drabowicz, 2017; Jones, Ramanau, Cross, y Healing, 2010; Kaznowska, Rogers y Usher, 2011; Margaryan et al., 2011; Ng, 2012; Prensky, 2011; Teo, Kabakçı Yurdakul, y Ursavaş, 2014; Thomas, 2011), por lo que no responderían a las definiciones clásica de un grupo etario particular, tales como Nativo Digital, New Millennium Learner, Millennials, entre otras denominaciones.

En el caso de los conocimientos y habilidades TIC de índole educativo, corresponden, principalmente a los desarrollados en su período escolar, asociados generalmente al uso del procesador de textos y el acceso a información en Internet (Hammond et al., 2009).

¿El uso de las TIC en la FIP podría generar cambios en la percepción del uso de las tecnologías en educación, y por consecuencia, el desarrollo de una actitud positiva hacia ellas? Según Larose, Grenon, Morin y Hasni (2009), serían las características de cada persona y no necesariamente lo que se realice durante la formación docente lo que produciría efectos positivos. Los autores observaron que los profesores que han utilizado las TIC durante su formación docente de manera estable y quienes las utilizaron esporádicamente no modifican sustancialmente el uso que hacen de ellas durante su labor docente. Guacaneme-Mahecha, Zambrano-Izquierdo y Gómez-Zermeño (2016) coinciden en reconocer la relevancia de la motivación o la experiencia del profesor al momento de incorporar las TIC.

Lo anterior manifiesta la necesidad de que las TIC estén presentes en el proceso formativo con un enfoque para el desarrollo de la actitud positiva. Esto se puede concretar con la creación de oportunidades de aprendizaje asociados a la integración didáctica de las TIC con una mirada integradora, las que deberían favorecer una mejor presencia de ellas en su futura práctica pedagógica. Estos cambios favorecerían el desarrollo de conocimientos y habilidades TIC que se encuentren relacionados con los usos de los futuros profesores.

Un constructo asociado a los conocimientos y habilidades TIC en un individuo es la noción de autoeficacia ${ }^{2}$, entendida en el contexto de este trabajo, como la creencia de que se

2 El concepto ha sido propuesto principalmente por Albert Bandura y abordado por otros autores (Covarrubias Apablaza y Mendoza Lira, 2013). 
es capaz de realizar tareas pedagógicas efectivas (que favorezcan el logro de aprendizajes ) utilizando las TIC (Albion, 1999; Hammond, Reynolds, e Ingram, 2011; Sang et al., 2010). La importancia de la autoeficacia radica en que puede influir en la aparición de una futura conducta por parte del individuo (Al-Awidi y Alghazo, 2012) y, por consecuencia, en una posible integración de las TIC en educación (Abbitt y Klett, 2007; Albion, 1999; Ertmer y Ottenbreit-Leftwich, 2010). Como consecuencia de lo anterior, podría producirse en el individuo un cambio en las creencias sobre la importancia de las TIC en educación.

Al respecto, un concepto bajo de autoeficacia en el uso de las TIC puede implicar estados de ansiedad o preocupación, lo contrario a quienes presentan un alto grado de ella (Sang et al., 2010). Lograr en los futuros profesores un concepto alto de autoeficacia frente al uso de las TIC implica que se produzcan experiencias exitosas con su uso en contextos educativos y, por tanto, un aumento en su uso (Kavanoz, Yüksel, y Özcan, 2015).

\subsubsection{Disciplina formativa}

Un segundo factor es la posible relación de las TIC con la disciplina formativa, sus posibilidades, dificultades y beneficios para la formación de profesores. Integrar didácticamente las TIC debería ser un proceso en el que se conjuguen los conocimientos sobre el uso de las TIC, conocimientos didácticos y conocimientos del contenido que posee un profesor (Koehler y Mishra, 2005). Es necesario, por tanto, conocer las herramientas TIC relacionadas con la disciplina que se enseña y que ayudarían a lograr aprendizajes en los estudiantes.

¿Existe relación entre la disciplina y la frecuencia o tipo de uso de las TIC que realiza un profesor? En una investigación sobre las diferencias en el uso de las TIC entre tres grupos de estudiantes: a) ciencias naturales, técnicas y de la computación; b) estudios sociales y de humanidades; y c) estudiantes del departamento de educación, Pšunder y Virtič (2010) concluyen que, debido a la naturaleza de sus disciplinas, los estudiantes del primer grupo son quienes poseen mayores conocimientos y habilidades TIC.

En Inglaterra, la Encuesta sobre Tecnologías de la Información y las Comunicaciones en las Escuelas (Department for Education Skills, 2003), evidenció que, dejando de lado la asignatura de Tecnología de la Información, las asignaturas en Educación Primaria que realizan un uso sustancial de las TIC son Inglés (Lenguaje), Matemáticas y Ciencias; y entre las que menor uso hacen de ellas están Educación Física, Lenguas modernas extranjeras y Ciudadanía. En el caso de la Educación Secundaria, el mayor uso está en Diseño y 
Tecnología; y Ciencias; y entre las de menor uso, se mantienen Educación Física y Ciudadanía, a las que se suman Humanidades y Educación Religiosa.

En la misma línea, los resultados chilenos de SITES 2008 (ENLACES y SITES, 2008) señalan que en $8^{\circ}$ año de Educación Básica (Primaria) el profesorado que más utiliza las TIC, es de Inglés u otra lengua extranjera, y el que menos lo hace es de Estudio y Comprensión de la Sociedad. Desde el punto de vista de los estudiantes (Collect y ENLACES, 2005), en Primaria el mayor uso se realizaría en Lenguaje y Comunicación y el menor en Educación Religiosa, y en Secundaria habría un mayor uso en Ciencias de la Naturaleza y un menor uso en Filosofía y Psicología.

En una encuesta realizada en 2012 a 601 profesores de Matemáticas, Lenguaje y Ciencias Naturales, se observan diferencias en el uso de las TIC en cuanto a la disciplina que se enseña: los profesores de Matemáticas y Ciencias Naturales triplican en frecuencia de uso a los profesores de lenguaje (Labbé, Matamala, Astudillo e Hinostroza, 2012).

La particularidad de cada disciplina podría implicar, por tanto, diferencias en el uso de las tecnologías, no solamente en la frecuencia de uso en el aula, sino principalmente desde la perspectiva de la aplicación pedagógica, es decir, el uso que se hace de ellas para el logro de aprendizajes. Por ejemplo, según Barton y Haydn (2006), los profesores de historia utilizarían mayormente software genérico o de uso pedagógico (que no ha sido diseñado para la enseñanza) y de visitas a sitios web específicos en sus clases, a diferencia de los profesores de ciencias, quienes tenderían a usar software específico para su asignatura, tal como software de simulación, de experimentación virtual, de modelado, entre otros.

Según Webb y Cox (2004), el profesorado usa las TIC principalmente para:

- simulación y modelado, en ciencia y otras asignaturas;

- ambientes de modelado y otro tipo de software en matemáticas;

- procesador de textos para lenguaje y literatura;

- uso de Internet para aumentar la información de la disciplina;

- software de presentaciones para desarrollar exposiciones por parte de los estudiantes y habilidades de alfabetización;

- pizarras interactivas para promover la discusión en clase y habilidades para explicar y presentar información.

El motivo de estas diferencias puede estar relacionado con lo señalado por Pšunder y Virtič (2010): el uso de las TIC difiere según su disciplina, pues cada una posee 
características y requerimientos que son propios. Si la enseñanza de una disciplina requiere utilizar simuladores, realizar experimentos virtuales, emplear telescopios $u$ otras herramientas se va configurando un tipo de uso de las tecnologías diferente al de alguien que se centra en la lectura de documentos o en la escritura en blogs.

Esto es fundamental para diseñar la adopción de las TIC en programas de formación de profesorado. El uso que se promueva de ellas a través de las distintas oportunidades de aprendizaje fomentará conocimiento y el desarrollo de cierto tipo de herramientas y de actividades. Esto requiere que los formadores conozcan distintos usos de las TIC y aprendan a utilizarlas para la enseñanza de sus disciplinas, favoreciendo la disposición para adoptarlas e integrarlas en sus clases (Escorcia-Oyola y Jaimes de Triviño, 2015; Gibson, Moline, y Dyck, 2011; Ottenbreit-Leftwich, Glazewski, Newby, y Ertmer, 2010).

\subsubsection{Creencias sobre el proceso de aprendizaje y su relación con las TIC}

Un tercer factor son las creencias pedagógicas del profesor, es decir, las concepciones sobre los mecanismos más apropiados para el logro del aprendizaje por parte de los estudiantes (Hermans et al., 2008). Por tanto, nos encontramos con propuestas teóricas que pueden orientar el uso de las TIC por parte del profesorado. A continuación, abordaremos desde distintas teorías la relevancia o pertinencia de incorporar las tecnologías en el proceso de enseñanza y aprendizaje, cuestión importante para entender el valor de las creencias sobre el proceso de aprendizaje para la integración y adopción de las TIC.

\subsubsection{Teorías sobre aprendizaje TIC}

Para comenzar, resaltamos una acotación que realizan Coll, Mauri y Onrubia:

Los profesores tienden a hacer usos de las TIC que son coherentes con sus pensamientos pedagógicos y su visión de los procesos de enseñanza y aprendizaje. Así, los profesores con una visión más transmisiva o tradicional de la enseñanza y del aprendizaje tienden a utilizar las TIC para reforzar sus estrategias de presentación y transmisión de los contenidos, mientras que los que tienen una visión activa o 'constructivista' tienden a utilizarlas para promover las actividades de exploración o indagación de los alumnos, el trabajo autónomo y el trabajo colaborativo. (2008, p. 83)

Es decir, la sola presencia de las tecnologías en el aula no asegura un aprendizaje profundo, sino que debe ir precedida de una intención de uso con sentido pedagógico y no 
meramente instrumental. Al mismo tiempo, la presencia de las TIC en el aula responde a una teoría o al menos una perspectiva asumida por el profesor sobre qué es la educación, sobre lo que implica el proceso de aprendizaje y, en particular, las cuestiones éticas, políticas o filosóficas implicadas (Siemens y Tittenberger, 2009).

Sobre los efectos de las TIC en educación, en primer lugar, es preciso señalar que la novedad que representa su en el aula, conlleva dudas sobre su real efectividad en el proceso de aprendizaje (Silva, Gros, Garrido y Rodríguez, 2006). Empero, la evidencia obtenida en diferentes investigaciones sugiere que se observan resultados, y se pueden traducir en un mayor aprendizaje por parte de los estudiantes, siempre siendo matizados o analizados a la luz de otras variables (Goldberg, Russell y Cook, 2003; Oteiza Morra, 2007).

Tal es el caso del The ICT Impact Report, una revisión de diecisiete estudios sobre el impacto de las TIC en escuelas europeas, en que se concluye que su incorporación reporta a la educación tanto beneficios en el aprendizaje como una percepción positiva de los profesores hacia ellas (Balanskat, Blamire, y Kefala, 2006). En un estudio de Marchesi y Martín (2003), se reporta una percepción positiva de los efectos de su presencia en el aula en aspectos como la interrelación, el ambiente de aula o la adaptación a las necesidades individuales, $\mathrm{Y}$ si bien los profesores consideraron que el aula tradicional permitía un mejor aprendizaje, los resultados académicos evidenciaban lo contrario.

En general, se aprecia que las diferencias positivas que conlleva el uso de la tecnología se encuentran relacionadas con las estrategias de aprendizaje empleadas. Es decir, las condiciones en que se produce un aprendizaje poseen un rol fundamental en la incorporación de las TIC en el aula. Por tanto, las creencias del profesor en torno a las características del proceso de aprendizaje se convierten en un elemento fundamental para el uso de las TIC. La creación de entornos de aprendizaje que sean propicios para el aprendizaje dependerá en definitiva de aquellas teorías, explícitas e implícitas, sobre cómo aprende el ser humano que fundamentan el trabajo del profesor (Guerrero y Flores, 2009).

\subsubsection{Enseñar con TIC y Enseñar las TIC}

El uso de la tecnología en la enseñanza por parte de los profesores responde a dos tendencias: enseñar con TIC y enseñar el uso de las TIC. La primera corresponde a la utilización de las TIC para lograr objetivos de aprendizaje del curriculum. La segunda, a la enseñanza específica del uso de una herramienta TIC. 
Los conocimientos y habilidades que aprenden los estudiantes -por ejemplo: en contexto escolar o educación superior- podrían ser transferidos a la vida personal y laboral futura. Lo que aprendan y la forma en que lo hagan tiene relevancia por sus consecuencias, y, por tanto, se necesita un profesorado preparado para su integración.

Para Jonassen (1999) existen tres fases en la historia del uso de los computadores en la enseñanza, identificados con el rol que se les concede: aprender de los ordenadores, aprender sobre los ordenadores y aprender con los ordenadores. De estos, la última fase representaría el paso a una tecnología que acompaña en el proceso de aprendizaje. Los profesores deben, por tanto, lograr una perspectiva del uso de las TIC que las identifiquen como:

- una ayuda a la construcción del conocimiento;

- una ayuda a la exploración de la información;

- una ayuda para el aprender haciendo;

- una ayuda al aprendizaje mediante la comunicación;

- compañeros intelectuales en pos del aprendizaje reflexivo.

Jonassen propone el uso de herramientas mentales (mindtools, en inglés), o herramientas computacionales. La función de éstas es doble: por una parte, facilitar el procesamiento cognitivo de los estudiantes (herramientas cognitivas); por otra parte, para que los estudiantes representen -o expresen- lo que saben, y no limitarse a reproducir lo que les dicen (Jonassen, 1999; 2006). Uno de los fundamentos en el trabajo del autor es el cambio conceptual. Existen varias investigaciones sobre este tema que sugieren que tales cambios involucran una profunda reestructuración de conceptos y formas de pensar (She y Liao, 2010). En el caso de los profesores y el uso de las TIC, resulta necesario crear el contexto que facilite este cambio conceptual.

Profundizando en ello, en cuanto un modelo es un sistema conceptual compuesto de elementos, relaciones, operaciones y reglas de interacción, que se expresan usando algún sistema externo de representación (Jonassen, 2006; Mayer, 2003), la formación del profesorado para la integración de las TIC debería ser capaz de dar a conocer o desarrollar un modelo concreto. De acuerdo a esta perspectiva, el proceso debería considerar tres pasos: el reconocimiento de un error, que impide que se pueda explicar la realidad; la construcción de un nuevo modelo, que dé explicación de la realidad; el uso del nuevo modelo, es decir, que se emplee para enfrentar un problema. 


\subsubsection{Creencias del profesorado sobre las TIC}

La creencia de que la presencia de las tecnologías pueden ser un aporte real al aprendizaje y que los estudiantes reaccionarán positivamente frente a ellas es fundamental para su integración (Hammond et al., 2009; Mumtaz, 2000; Ottenbreit-Leftwich et al., 2010) ¿Qué fundamenta esta creencia? Una tercera variable para el desarrollo de la $A(T I C)+y$ $\mathrm{A}(\mathrm{IntDid})+\mathrm{y}$, por tanto, para la integración de las tecnologías en los procesos de aprendizaje, corresponde a las creencias sobre cómo se logra aprendizajes empleando las TIC. Este factor no es menor pues la eficacia de las TIC en el aula es mucho mayor tanto en cuanto existe coherencia entre ellas y las creencias pedagógicas que posee un profesor (AvidovUngar y Eshet-Alkakay, 2011; Gobierno de Chile, 2009; Salleh, 2016).

Ottenbreit-Leftwich et al. (2010) destacan que cuando un profesor se enfrenta a una innovación que le es propuesta establece juicios de valor, los que influyen en la aceptación o no de ella y que esto es especialmente reconocible en el caso de las TIC. En cuanto las creencias no cambian tan fácilmente, su influencia es importante en la construcción de juicios de valor y estos perduran en cuanto se mantienen la creencia que lo sustenta. Por ello, el uso de tecnologías durante la FIP y la adquisición de conocimientos y habilidades TIC no producirán cambios positivos por sí mismos en la implementación de las tecnologías si no son coherentes con el sistema de creencias bajo el que se está formando el profesor. Así, por cuanto se van construyendo creencias sobre cuestiones pedagógicas durante la formación docente en las experiencias formativas que se realizan (Markauskaite, 2007) y las experiencias profesionales que desarrollan (Area Moreira, 2004), es de especial interés que los formadores atiendan esta cuestión.

En general, los profesores que tienen un sistema de creencias constructivista tienden a utilizar más las tecnologías que los profesores con una visión tradicional, tienen mayor conciencia de los beneficios de las TIC para los aprendizajes y realizan un uso más avanzado de ellas (Ertmer y Ottenbreit-Leftwich, 2010; Hermans et al., 2008; Jimoyiannis y Komis, 2007; Mumtaz, 2000; Petko, 2012; Sang et al., 2010). Esto no significa que el segundo grupo de profesores no las utilice, sino que la diferencia se encuentra en el tipo de uso que hacen de ellas (Tondeur, Hermans, van Braak, y Valcke, 2008). Esta relación entre creencia constructivista sobre la educación y uso de las TIC es importante pues:

Cuando la mediación pedagógica no se sostiene dentro de la perspectiva constructivista, las TIC se convierten en meros transmisores o reproductores de contenidos predefinidos por los docentes quienes no asumen los saberes previos de 
los aprendices, no atienden las necesidades ni características particulares y propias de los que aprenden, como tampoco el contexto en el cual ocurren los aprendizajes buscados (Román, 2010, p. 110)

Todo este proceso supone que se produzca un cambio en el profesor, el que se puede lograr en un contexto que se haya diseñado para integrar las TIC en experiencias formativas - oportunidades de aprendizaje- y administrativas.

\subsection{Propuestas}

El proceso de adopción e integración de las TIC por parte del profesorado en sus prácticas pedagógicas no se resuelve con la incorporación de tecnologías en la FIP, pero este proceso sí permitiría desarrollar una actitud positiva hacia las TIC y hacia su integración didáctica. Tal proceso de adopción (cambio) e integración resulta complejo y debe abordarse en sus variadas aristas y particularidades, de forma tal que la formación docente sea capaz de promover el uso de las TIC por parte de los futuros profesores.

En los siguientes párrafos estructuraremos, a partir de dos modelos teóricos, diversas propuestas para el desarrollo de la actitud positiva, mencionando la estructura formativa más adecuada, explicitando propuestas de oportunidades de aprendizaje, la vinculación con el

prácticum, y los contenidos de la formación en tecnologías, tanto de la disciplina formativa con para el uso general.

\subsubsection{Desarrollar las actitudes en la formación pre-service: IMTPG y TPACK}

Tradicionalmente, nos señala Guskey (2002), se ha entendido el proceso de desarrollo docente como resultado de una serie de cambios, de forma que una modificación en los conocimientos y creencias produciría cambios en la conducta en el aula y, como consecuencia, modificación en los resultados del proceso de enseñanza-aprendizaje. No obstante, el autor considera que son los resultados de aprendizaje produciría un cambio en las creencias sobre lo que resulta efectivo -y lo que no-, y tales cambios son resultado de modificaciones en las prácticas en el aula. Trasladando este modelo hacia la formación en el uso de las TIC, los resultados de aprendizaje tras su integración supondrían la construcción de creencias en los futuros profesores, y por consecuencia, de actitudes hacia la integración de las TIC en educación. Por tanto, contar con oportunidades de aprendizaje efectivas con uso de las TIC y reflexionar sobre ellas facilitaría la construcción de una visión sobre las 
posibilidades y desafíos que para el aprendizaje conllevan las TIC.

Un modelo que fundamenta un proceso como el mencionado es el Interconnected Model of Teacher Professional Growth (IMTPG o IM), de Clarke y Hollingsworth (2002). Este modelo (ver Figura 2) considera la existencia de cuatro dominios que constituyen el espacio de desarrollo del profesor. Estos dominios cambian a partir de un proceso de activación y reflexión, y considera la puesta en práctica de las creencias, conocimientos o prácticas del profesor. Este modelo puede ser útil para analizar y entender el proceso de cambio personal, tanto como para diseñar un proyecto de formación profesional (Coenders y Terlouw, 2015; Justi y van Driel, 2006), pues un cambio en uno de los dominios repercute en el resto.

Figura 2

Esquema del modelo IMTPG

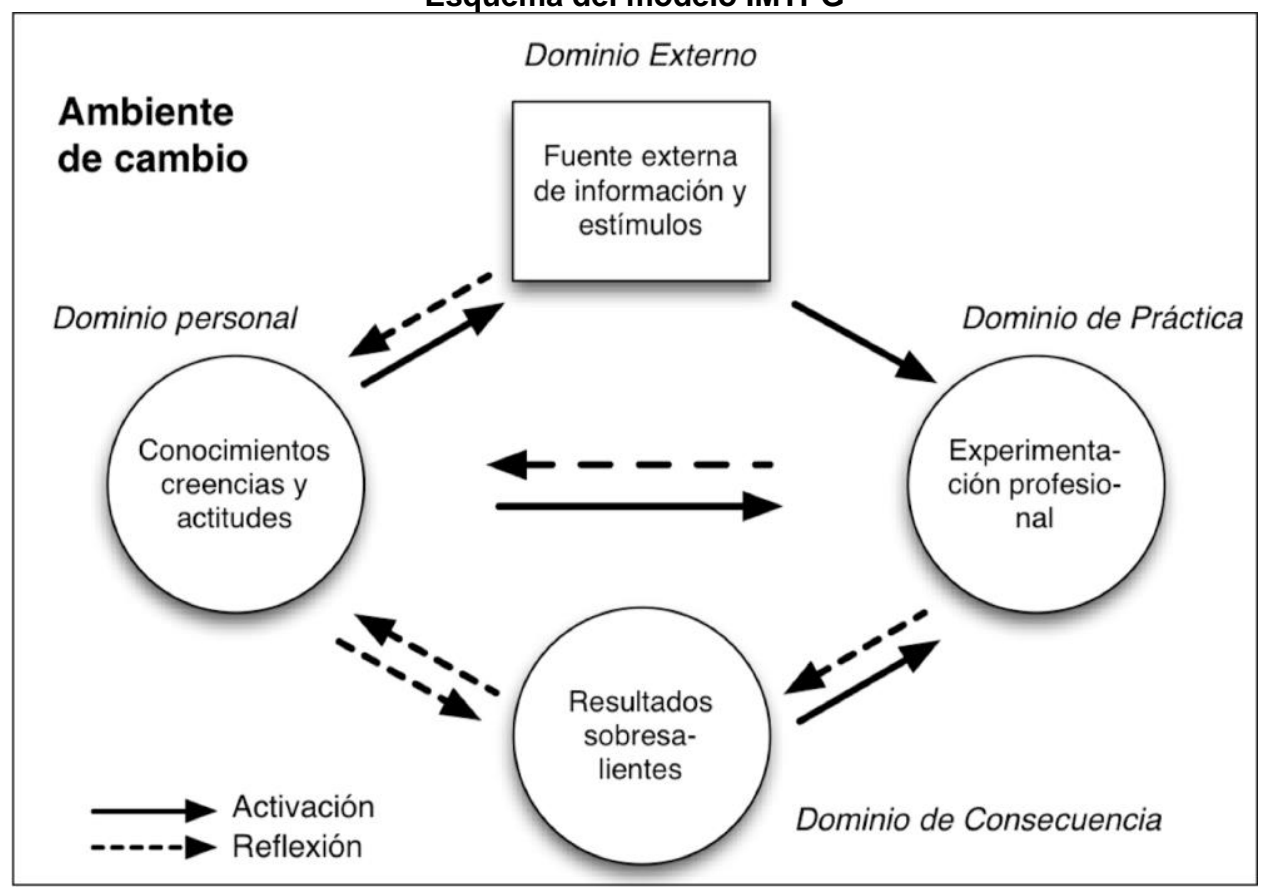

Fuente: Adaptación propia a partir de Clarke (2002)

Por ejemplo, las experiencias nuevas de formación -correspondientes al dominio de la práctica- conllevarían cambios en los conocimientos y creencias y, por tanto, posibles cambios en las actitudes hacia las TIC en contextos educativos. O una $A(\operatorname{IntDid})+$, que lleve a integrar las TIC en sus prácticas educativas y produzca aprendizajes profundos en los estudiantes, traerá como consecuencia la consolidación de la práctica.

Un segundo modelo que destacamos es el Technological pedagogical content knowledge (TPACK, en adelante), el cual relaciona tres conocimientos profesionales que 
enmarcan el actuar docente y que deberían ser abordados durante la FIP. Dichos conocimientos son el Conocimiento Tecnológico, el Conocimiento Pedagógico y el Conocimiento del Contenido. De la interacción entre estos conocimientos surge el Conocimiento Pedagógico del Contenido, Conocimiento Tecnológico Didáctico, el Conocimiento Tecnológico del Contenido y el Conocimiento Tecnológico Didáctico del Contenido (Jamieson-Proctor, Finger, y Albion, 2010; Koehler, Mishra, y Cain, 2015; Koh y Chai, 2016). Cada uno de estos componentes debe fundamentar la formación y ser integrado, de forma particular y en conjunto. Por tanto, el desarrollo de las actitudes positivas supone crear oportunidades de aprendizaje contextualizadas, fundamentadas y centradas en el profesor en formación. A continuación, esbozamos algunas ideas coherentes con esta propuesta ${ }^{3}$.

\subsubsection{Estructura formativa para la formación en TIC}

Las oportunidades de aprendizaje que favorezcan fundamentar e integrar las TIC se pueden incorporar en la formación inicial de profesores mediante alguna de las siguientes estructuras:

i. Incorporación de asignatura(s) específica(s) para la Formación en TIC. Por ejemplo, Enseñanza de matemática con TIC.

ii. Formación para la integración de las TIC en varias o todas las asignaturas del programa de formación, dotándole la formación TIC de un carácter transversal.

iii. Formación Mixta. Se obtienen los beneficios de las dos estrategias anteriores: la visión transversal de la integración de las TIC y la formalización de los conocimientos y habilidades que permiten una asignatura específica.

Un desafío asociado con el logro de los enfoques es considerar los conocimientos y creencias previas de los estudiantes sobre las TIC (Lee y Kim, 2014). Consideramos que la estructura formativa mixta podría propiciar el desarrollo de actitudes positivas en cuanto favorece la presencia práctica de las TIC en diversos procesos de aprendizaje, con enfoque particular según la asignatura que se curse y las características del formador. $Y$, al mismo tiempo, se puede potenciar la sistematización y formalización de los conocimientos y habilidades desde una mirada concreta en el transcurso de una asignatura específica sobre TIC en educación.

\footnotetext{
${ }^{3}$ Algunas de las cuestiones siguientes han sido abordadas en Tapia Silva (2017)
} 


\subsubsection{Oportunidades de aprendizaje}

Se debe propender a la integración constante de las TIC en las estrategias de formación del profesor, diseñando oportunidades de aprendizaje que integren las TIC en las diversas asignaturas del programa formativo considerando, como señalan Brush y Saye (2009), tres premisas orientativas:

- Ver, criticar y discutir experiencias de profesores que utilizan las TIC.

- Proporcionar oportunidades para conocer tecnologías innovadoras, cómo se integran en su propia formación docente, y que después puedan emplear en las clases.

- Proporcionar oportunidades para implementar actividades que utilicen efectivamente las TIC en las aulas.

Bajo estas premisas se puede orientar el diseño de oportunidades de aprendizaje, las que pueden variar, dependiendo de los diversos factores del contexto (Haydn, 2010). Entre las oportunidades de aprendizaje destacamos:

a. Análisis de buenas prácticas de integración de las TIC en salas de clase, tarea que es realizada por formadores y futuros profesores, a fin de identificar los aspectos que caracterizarían un uso efectivo de las TIC (Brush y Saye, 2009). Esta actividad permitirá identificar, por ejemplo, posibles relaciones entre conocimientos disciplinares y pedagógicos: ¿qué se necesita para poner en práctica la estrategia y en qué aspectos debe enfocarse el profesor? ¿Qué estrategias empelar para representar un tema específico? ¿Cuál es el nivel de cumplimiento de las políticas educativas TIC? ¿Cómo se evalúa el trabajo realizado con TIC?

b. Trabajo en actividades específicas con uso de la tecnología. Se deben diseñar oportunidades de aprendizaje que les permitan adoptar e integrar las TIC y que consideren diversas herramientas. Una posibilidad es el trabajo con el modelo de Tipos de Actividades desarrollado por Harris y Hofer (Harris y Hofer, 2009; Harris, Mishra, y Koehler, 2009; Harris, 2011; Hofer y Harris, 2011).

\subsubsection{TIC y Prácticum}

El prácticum tiene por objetivo aplicar y evaluar en un contexto escolar las competencias desarrollados durante la FIP a través de la observación, ayudantía, planificación, aplicación, entre otras actividades. En este contexto, se podrían identificar las posibilidades y desafíos de las TIC para el aprendizaje (McDougall, 2008), a pesar de las dificultades que se presenten: escasez de recursos en el establecimiento educativo, falta de 
conocimientos y habilidades TIC en profesores guías, coordinadores académicos, u otros, o pocas oportunidades concretas para poder integrar TIC en el aula (Brush y Saye, 2009; Niess, 2005; 2008).

Se propone, por tanto, diseñar oportunidades de aprendizaje coherentes con el proceso en que se encuentre el estudiante que les permitan profundizar en el desarrollo de conocimientos, habilidades y una actitud positiva hacia su integración didáctica -A(IntDid)+-. Por ejemplo:

- Crear portafolio virtual o plataforma online para recoger evidencia, registrar la reflexionar y comunicarse con supervisor y compañeros (Cebrián de la Serna, 2011).

- Investigar sobre prácticas pedagógicas que integren las TIC llevadas a cabo por profesores guías.

- Diseñar e implementar intervenciones didácticas integrando las TIC para el logro de un objetivo de aprendizaje.

- Diseñar, crear o emplear recursos educativos que consideren el uso de TIC, que respondan a una complejidad cognitiva variable, y posibles de usar tanto por el formador como por el uso del profesor del centro educativo.

\subsubsection{Formación en tecnologías de la disciplina formativa y de uso general}

Un conocimiento base en un profesor es el conocimiento de la disciplina; y en cuanto cada disciplina cuenta con una serie de tecnologías asociadas a su desarrollo, investigación o tareas, se pueden lograr conocimientos y habilidades tecnológicas que quienes se dedican específicamente a la disciplina poseen. Por ejemplo, el uso de microscopios en la investigación en biología, telescopios en astronomía, procesadores de texto para escritores o periodistas, planillas de cálculo por parte de ingenieros o contadores, documentos digitalizados por parte de historiadores, editores de imágenes por parte de diseñadores, entre otros.

Es importante, por tanto, que el futuro profesor conozca y emplee herramientas digitales propias de su disciplina a enseñar, las que podrían ser utilizadas durante las asignaturas disciplinares. Se debe tener, en este ámbito, especial cuidado al realizar el modelado del uso de las distintas herramientas tecnológicas. Por ejemplo, no sólo centrarse en el logro del aprendizaje del contenido curricular, sino también explicitar la evaluación del uso de la herramienta, por ejemplo, solicitando que se expliquen los procedimientos de uso de ella. 
En conjunto con el conocimiento de las herramientas tecnológicas propias de la disciplina, se deben promover el empleo de herramientas tecnológicas de uso general procesador de textos, editor de vídeos, cámara fotográfica- para diseñar y construir productos que necesiten emplear en sus clases (Jamieson-Proctor et al., 2010), evaluando las posibilidades y limitaciones que conlleva el uso de una herramienta (Niess, 2008).

Estos dos enfoques formativos -aprender a utilizar tecnologías específicas de la disciplina y aprender a utilizar tecnologías de uso general- son complementarios y favorecerían que los futuros profesores desarrollen conocimientos y habilidades en el uso de las TIC. Por una parte, estos aumentarían en el uso de las herramientas tecnológicas y, por tanto, la confianza en su uso de las mismas, aportando al desarrollo del A(TIC)+. Por otra parte, el conocimiento de los procedimientos de uso favorecería su integración para el diseño de intervenciones didácticas, permitiendo desarrollar A(IntDid)+.

\section{Síntesis y reflexiones finales}

El constructo Actitud hacia las TIC se puede comprender desde una predisposición o tendencia hacia las TIC como objeto de la Actitud y, por otra parte, hacia la integración didáctica de las TIC. Se han denominado $A(T I C)$ y $A($ IntDid), respectivamente, y pueden ser tanto positiva (a favor) como negativa (en contra).

La complejidad operativa del constructo Actitud hacia las TIC -en sus dos acercamientos mencionados- tiene su origen en los diversos factores que intervienen en su construcción. Tres de los factores que intervienen en la construcción de una actitud son a) los Conocimientos y habilidades TIC; b) la Disciplina formativa en que se especializan los profesores; y c) Creencias sobre el proceso de aprendizaje y su relación con las TIC.

Para el desarrollo de una Actitud positiva hacia las TIC, es importante diseñar y ejecutar oportunidades de aprendizaje que les permitan a los profesores en formación inicial aprender tecnologías y, por tanto, aumentar los conocimientos y habilidades relativos a su uso e integración. Para ello, se debe considerar la presencia de objetivos formativos específicos relacionados con la integración de las TIC, los que deberían estar presentes en asignaturas concretas y abordados de manera transversal en diversas asignaturas.

En segundo lugar, la implementación de las oportunidades de aprendizaje para desarrollar los conocimientos y habilidades TIC debería buscar dos cuestiones esenciales: a) análisis de experiencias efectivas (por ejemplo, estudios de caso) en los que se observe el uso de la tecnología en salas de clase y se analice; y b) diseño de oportunidades de 
aprendizaje en las cuales los profesores en formación adopten el rol de estudiantes y se modele la integración de TIC. De manera complementaria, en tercer lugar, las oportunidades de aprendizaje para el uso de las TIC podrían estar presentes en el prácticum a fin de darle un contexto real de aplicación y evaluación de las tecnologías, relacionado tanto con lo que hacen los estudiantes practicantes como los profesores guías del prácticum.

Se destaca la importancia del conocimiento concreto de herramientas tecnológicas, tanto las que son empleadas por quienes ejercen la disciplina, como aquellas herramientas que poseen un carácter general. El aumento del conocimiento y habilidades TIC debería implicar, junto a los cambios en las estrategias y los momentos formativos, una serie de cambios personales y en los resultados del trabajo.

En última instancia, mencionamos algunas condiciones que pueden dificultar el desarrollo de una actitud positiva hacia las TIC y su integración didáctica. Ente ellas se encontrarían las actitudes hacia las TIC de los formadores de futuros profesores; las condiciones materiales o institucionales asociadas al proceso formativo en TIC y los conocimientos y habilidades TIC que se consideran en el currículum formativo.

\section{Referencias}

Abbitt, Jason. (2011). An Investigation of the Relationship between Self-Efficacy Beliefs about Technology Integration and Technological Pedagogical Content Knowledge (TPACK) among Preservice Teachers. Journal of Digital Learning in Teacher Education, 27(4), 134-143.

Recuperado

de http://www.eric.ed.gov/ERICWebPortal/contentdelivery/servlet/ERICServlet?accno=EJ9 36541

Abbitt, Jason T., y Klett, Mitchell. (2007). Identifying influences on attitudes and self-efficacy beliefs towards technology integration among pre-service educators. Electronic Journal for the Integration of Technology in Education, 6, 28-42.

Agyei, Douglas y Voogt, Joke. (2011). Exploring the potential of the will, skill, tool model in Ghana: Predicting prospective and practicing teachers' use of technology. Computers \& Education, 56(1), 91-100. doi:10.1016/j.compedu.2010.08.017

Al-Awidi, Hamed Mubarak y Alghazo, Iman Mohammad. (2012). The effect of student teaching experience on preservice elementary teachers' self-efficacy beliefs for technology integration in the UAE. Educational Technology Research and Development, 60(5), 923-941. doi:10.1007/s11423-012-9239-4

Albion, Peter. (1999). Self-efficacy beliefs as an indicator of teachers' preparedness for teaching with technology. 10th International Conference of the Society for Information Technology \& Teacher Education (SITE 1999), 28 febr - 4 mar, 1602-1608. Recuperado de http://eprints.usq.edu.au/6973/ 
Albirini, Abdulkafi. (2006). Teachers' attitudes toward information and communication technologies: the case of Syrian EFL teachers. Computers \& Education, 47(4), 373-398. doi:10.1016/j.compedu.2004.10.013

Amelii, María Rita, Reyes, Ana María y Ríos Colmenárez, María Janeth. (2017). Las TIC en la Formación Inicial Docente en América Latina. Tendencias Innovación en la Sociedad Digital, 1(1), 27-39. Recuperado de http://saber.ucv.ve/ojs/index.php/rev_TISD/article/view/14593

Area Moreira, Manuel. (2004). Los medios y las tecnologías en la educación. Madrid: Pirámide.

Avidov-Ungar, Orit, y Eshet-Alkakay, Yoram. (2011). Teachers in a World of Change: Teachers' Knowledge and Attitudes towards the Implementation of Innovative Technologies in Schools. Interdisciplinary Journal of E-Learning and Learning Objects, 7(1), 291-303.

Balanskat, Anja, Blamire, Roger y Kefala, Stella. (2006). The ICT impact report. A review of studies of ICT impact on schools in Europe. Comunidades Europeas. Recuperado de http://unpan1.un.org/intradoc/groups/public/documents/unpan/unpan037334.pdf

Barton, Roy y Haydn, Terry. (2006). Trainee teachers' views on what helps them to use information and communication technology effectively in their subject teaching. Journal of Computer Assisted Learning, 22(4), 257-272. Recuperado de http://onlinelibrary.wiley.com/doi/10.1111/j.1365-2729.2006.00175.x/abstract

Borkowski, Nancy. (2005). Organizational behavior in health care. Estados Unidos de América: Jones \& Bartlett Learning.

Brun, Mario. (2011). Las tecnologías de la información y las comunicaciones en la formación inicial docente de América Latina (Políticas sociales, 172). Recuperado de https://repositorio.cepal.org/bitstream/handle/11362/6183/1/S1100626_es.pdf

Brush, Thomas y Saye, John. (2009). Strategies for Preparing Preservice Social Studies Teachers to Effectively Integrate Technology: Models and Practices. Contemporary Issues in Technology and Teacher Education, 9(1), 46-59. Recuperado de http://editlib.org/p/28300

Calvani, Antonio, Fini, Antonio, Ranieri, Maria, y Picci, Patrizia. (2012). Are young generations in secondary school digitally competent? A study on Italian teenagers. Computers \& Education, 58(2), 797-807. doi:10.1016/j.compedu.2011.10.004

Castro, Santiago, Guzmán, Belkys y Casado, Dayanara. (2007). Las TIC en los procesos de enseñanza y aprendizaje. Laurus, 13(23). Recuperado de http://www.redalyc.org/html/761/76102311

Cebrián de la Serna, Manuel. (2011). Supervisión con e-portafolios y su impacto en las reflexiones de los estudiantes en el Practicum. Estudio de caso. Revista de Educación, (354), 183-208. 
Cheung, Derek. (2009). Developing a Scale to Measure Students' Attitudes toward Chemistry Lessons. International Journal of Science Education, 31(16), 2185-2203. doi:10.1080/09500690802189799

Clark, Sarah y Byrnes, Deborah. (2015). What Millennial Preservice Teachers Want to Learn in Their Training. Journal of Early Childhood Teacher Education, 36(4), 379-395. doi:10.1080/10901027.2015.1100148

Clarke, David y Hollingsworth, Hilary. (2002). Elaborating a model of teacher professional growth. Teaching and Teacher Education, 18(8), 947-967. doi:10.1016/s0742$051 \times(02) 00053-7$

Coenders, Fer y Terlouw, Cees. (2015). A Model for In-service Teacher Learning in the Context of an Innovation. Journal Science Teacher Education, 26(5), 451-470. doi:10.1007/s10972-015-9432-5

Coll, César, Mauri, Teresa, y Onrubia, Javier. (2008). La utilización de las tecnologías de la información y la comunicación en la educación: Del diseño tecno-pedagógico a las prácticas de uso. En César Monereo (Ed.), Psicología de la educación virtual: aprender y enseñar con las tecnologías de la información y la comunicación (pp. 74-103). Madrid: Morata. Recuperado de http://portales.puj.edu.co/javevirtual/portal/documentos/psicologia_de_la_educacion_virt ual.pdf

Collect, y ENLACES. (2005). Encuesta "Educación en la Sociedad de la Información". Santiago de Chile: ENLACES. Recuperado de http://www.observatoriodigital.gob.cl/sites/default/files/encuesta_educacion_en_la_soci edad_de_la_informacion_2005.pdf

Covarrubias Apablaza, Carmen G. y Mendoza Lira, Michelle. (2013). La teoría de autoeficacia y el desempeño docente: El caso de Chile. Estudios hemisféricos y polares, 4(2), 107-123.

Department for Education Skills. (2003). Survey of Information and Communications Technology in Schools 2003 (Internet only). Recuperado de http://dera.ioe.ac.uk/4796/1/bweb05-2003.pdf

Drabowicz, Tomasz. (2017). Social theory of internet use: Corroboration or rejection among the digital natives? Correspondence analysis of adolescents in two societies. Computers \& Education, 105, 57-67. doi:10.1016/j.compedu.2016.10.004

Drossel, Kerstin, Eickelmann, Birgit y Gerick, Julia. (2017). Predictors of teachers' use of ICT in school - the relevance of school characteristics, teachers' attitudes and teacher collaboration. Education and Information Technologies, 22(2), 551-573. doi:10.1007/s10639-016-9476-y

Dunn, Karee E. y Rakes, Glenda C. (2011). Teaching teachers: An investigation of beliefs in teacher education students. Learning Environments Research, 14(1), 39-58. doi:10.1007/s10984-011-9083-1 
ENLACES, \& SITES. (2008). Second Information Technology and Education Study - SITES 2006. Resultados Nacionales SITES 2006. Recuperado de http://docplayer.es/4544218-Second-information-technology-and-education-study-sites2006-resultados-nacionales-sites-2006.html

Enochsson, Ann-Britt, y Rizza, Caroline. (2009). ICT in Initial Teacher Training: Research Review. Recuperado de http://www.oecd-ilibrary.org/education/ict-in-initial-teachertraining-research-review_220502872611

Ertmer, Peggy A, y Ottenbreit-Leftwich, Anne T. (2010). Teacher technology change: how knowledge, confidence, beliefs, and culture Intersect. Journal of Research on Technology in Education, 42(3), 255-284. Recuperado de https://files.eric.ed.gov/fulltext/EJ882506.pdf

Escofet Roig, Anna, García Gonzalez, lolanda, y Gros Salvat, Begoña. (2011). Las nuevas culturas de aprendizaje y su incidencia en la educación superior. Revista mexicana de investigación educativa, 16(51), 1177-1195. Recuperado de http://www.scielo.org.mx/scielo.php?pid=S1405-66662011000400008\&script=sci_arttext

Escorcia-Oyola, Ludmila, y Jaimes de Triviño, Clara. (2015). Tendencias de uso de las TIC en el contexto escolar a partir de las experiencias de los docentes. Educación y Educadores, 18(1), 137-152. Recuperado de http://www.redalyc.org/html/834/83439194008

Gibson, Susan, Moline, Teddy y Dyck, Brenda. (2011). What are education students' perceptions of the role of technology in social studies pedagogy? Alberta Journal of Educational Research, 57(1), 73-87. Recuperado de http://ajer.journalhosting.ucalgary.ca/index.php/ajer/article/viewFile/870/814

Goldberg, Amie, Russell, Michael y Cook, Abigail. (2003). The effect of computers on student writing: A meta-analysis of studies from 1992 to 2002. Journal of Technology, Learning, and Assessment, 2(1), 1-52. Recuperado de https://ejournals.bc.edu/ojs/index.php/jtla/article/view/1661

Gobierno de Chile, Fondo de Investigación y Desarrollo en Educación. (2009). Proyecto Factores que inciden en el desarrollo y sustentabilidad de prácticas innovadoras de integración curricular de tecnologías de la información (TICs) en la sala de clases en profesores de Enseñanza Media. Santiago de Chile: MINEDUC.

González Pérez, Alicia y De Pablos Pons, Juan. (2015). Factores que dificultan la integración de las TIC en las aulas. Revista de Investigación Educativa, 33(2), 401. doi:10.6018/rie.33.2.198161

Guacaneme-Mahecha, Marisol, Zambrano-Izquierdo, David y Gómez-Zermeño, Marcela Georgina. (2016). Apropiación tecnológica de los profesores: el uso de recursos educativos abiertos. Educ.Educ., 19(1), 105-117. doi:10.5294/edu.2016.19.1.6

Guerrero, Tivisay y Flores, Hazel. (2009). Teorías del aprendizaje y la instrucción. Educere: Revista Venezolana de Educación, 13(45), 317-329. 
Guitart Aced, Ros. (2002). Las actitudes en el centro escolar: reflexiones y propuestas. Barcelona: Editorial Graó.

Guskey, Thomas R. (2002). Professional development and teacher change. Teachers and Teaching: theory and practice, 8(3/4), 381-391. doi:10.1080/13540600210000051

Guzman, Angélica y Nussbaum, Miguel. (2009). Teaching competencies for technology integration in the classroom: Technology integration in the classroom. Journal of Computer Assisted Learning, 25(5), 453-469. doi:10.1111/j.1365-2729.2009.00322.x

Haddock, Geoffrey y Huskinson, Thomas L. H. (2004). Individual differences in attitude structure. En Geoffrey Haddock and Gregory R. Maio (Eds.), Contemporary perspectives on the psychology of attitudes (pp. 35-56). Hove, East Sussex; New York: Psychology Press.

Hammond, Michael, Crosson, Sue, Fragkouli, Elpiniki, Ingram, Jennifer, Johnston Wilder, Peter, Johnston Wilder, Sue, Kingston, Yvette, Pope, Melanie, Wray, David. (2009). Why do some student teachers make very good use of ICT? An exploratory case study. Technology, Pedagogy and Education, 18(1), 59-73. doi:10.1080/14759390802704097

Hammond, Michael, Reynolds, Lawrence, y Ingram, Jennifer. (2011). How and why do student teachers use ICT? Journal of Computer Assisted Learning, 27(3), 191-203. doi:10.1111/j.1365-2729.2010.00389.x

Harris, Judith. (2011). Activity Types Wiki. Recuperado de http://activitytypes.wm.edu

Harris, Judith y Hofer, Matthew. (2009). Instructional planning activity types as vehicles for curriculum-based TPACK development. En C. D. Maddux (Ed.), Research Highlights in Technology and Teacher Education (pp. 99-108). Estados Unidos de América: SITE.

Harris, Judith, Mishra, Punya y Koehler, Matthew (2009). Teachers' technological pedagogical content knowledge and learning activity types: Curriculum-based technology integration reframed. Journal of Research on Technology in Education, 41(4), 393-416. Recuperado de http://www.eric.ed.gov/ERICWebPortal/contentdelivery/servlet/ERICServlet?accno=EJ8 44273

Haydn, Terry. (2010). Case studies of the ways in which initial tracher training providers in England prepare student to use ICT effectively in their subject teaching. Norwich: CERI/OCDE. Recuperado de https://www.oecd.org/edu/ceri/45046837.pdf

Henerson, Marlene, Morris, Lynn Lyons, y Fitz-Gibbon, Carol Taylor. (1987). How to measure attitudes. Newbury Park, Calif.: Sage Publications.

Hermans, Ruben, Tondeur, Jo, van Braak, Johan y Valcke, Martín. (2008). The impact of primary school teachers' educational beliefs on the classroom use of computers. Computers \& Education, 51(4), 1499-1509. doi:10.1016/j.compedu.2008.02.001

Hinostroza, Enrique. (2017). TIC, educación y desarrollo social en América Latina y el Caribe. Recuperado de http://unesdoc.unesco.org/images/0026/002628/262862s.pdf 
Hofer, Matthew y Harris, Judith. (2011). Tipos de actividades de aprendizaje en el área de Ciencias Sociales. Recuperado de https://activitytypes.wmwikis.net/file/view/SocialStudiesLearningATs-

Feb2011Spanish.pdf/353468790/SocialStudiesLearningATs-Feb2011Spanish.pdf

ISTE (International Society for Tecnology in Education). (2008). NETS for Teachers 2008. Recuperado de http://www.iste.org/docs/pdfs/nets-t-standards.pdf?sfvrsn=2

Jamieson-Proctor, Romina, Finger, Glenn y Albion, Peter. (2010). Auditing the TK and TPACK confidence of pre-service teachers: Are they ready for the profession? Australian Educational Computing, 25(1), 8-17. Recuperado de http://acce.edu.au/journal/25/1/auditing-tk-and-tpack-confidence-pre-service-teachersare-they-ready-profession

Jimoyiannis, Athanassios y Komis, Vassilis. (2007). Examining teachers' beliefs about ICT in education: Implications of a teacher preparation rogramme. Teacher Development, 11(2), 149-173. doi:10.1080/13664530701414779

Johnson, Kristine. (2006). The millennial teacher: Metaphors for a new generation. Pedagogy, 6(1), 7-24.

Jonassen, David H. (1999). Computers as Mindtools for Schools: Engaging Critical Thinking. New Mexico, Estados Unidos: Prentice Hall.

Jonassen, David H. (2006). Modeling with Technology: Mindtools for Conceptual Change. Upper Saddle River, N.J: Pearson Merrill Prentice Hall.

Jones, Chris, Ramanau, Ruslan, Cross, Simon, y Healing, Graham. (2010). Net generation or Digital Natives: Is there a distinct new generation entering university? Computers \& Education, 54(3), 722-732. doi:10.1016/j.compedu.2009.09.022

Jung, Insung. (2005). ICT-Pedagogy integration in teacher training: application cases worldwide. Educational Technology \& Society, 8(2), 94-101.

Justi, Rosária y van Driel, Jan. (2006). The use of the Interconnected Model of Teacher Professional Growth for understanding the development of science teachers' knowledge on models and modelling. Teaching and Teacher Education, 22(4), 437-450. doi:10.1016/j.tate.2005.11.011

Kavanoz, Suzan, Yüksel, H. Gülru y Özcan, Emrah. (2015). Pre-service teachers' selfefficacy perceptions on Web Pedagogical Content Knowledge. Computers \& Education, 85, 94-101. doi:10.1016/j.compedu.2015.02.005

Kaznowska, Edyta, Rogers, Jason, y Usher, Alex. (2011). The State of E-Learnin in Canadian Universities, 2011: If Students Are Digital Natives, Why Don't They Like E-Learning? Recuperado de https://higheredstrategy.com/wpcontent/uploads/2011/09/InsightBrief42.pdf 
Kim, Jong Hye, Jung, Soon Young y Lee, Won Gyu. (2008). Design of contents for ICT literacy in-service training of teachers in Korea. Computers \& Education, 51(4), 16831706. doi:10.1016/j.compedu.2008.05.001

Knezek, Gerald, y Christensen, Rhonda. (2008). The importance of Information Technology attitudes and competencies in primary and secondary education. En Joke Voogt and Gerald Knezek (Eds.), International handbook of information technology in primary and secondary education (Vol. 1, pp. 321-331). Estados Unidos de América: Springer US.

Koehler, Mathew J., y Mishra, Punya. (2005). What happens when teachers design educational technology? The development of technological pedagogical content knowledge. Journal of educational computing research, 32(2). Recuperado de http://journals.sagepub.com/doi/pdf/10.2190/0EW7-01WB-BKHL-QDYV

Koehler, Matthew, Mishra, Punya, y Cain, William. (2015). ¿Qué son los Saberes Tecnológicos y Pedagógicos del Contenido (TPACK). Virtualidad, Educación y Ciencia, 6(10), 9-23. Recuperado de https://revistas.unc.edu.ar/index.php/vesc/article/download/11552/11983

Koh, Joyce Hwee Ling y Chai, Ching Sing. (2016). Seven design frames that teachers use when considering technological pedagogical content knowledge (TPACK). Computers \& Education, 102, 244-257. doi:10.1016/j.compedu.2016.09.003

Kroenung, Julia y Bernius, Steffen. (2012). Four Types of Attitudes in ICT Acceptance and Use? A Critical Assessment on the Basis of Empirical and Scientometric Data. Proceedings from 45th Hawaii International Conference on System Sciences (HICSS).

Labbé, Christian, Matamala, Carolina, Astudillo, Gustavo e Hinostroza, J. Enrique. (agosto, 2012). Uso de TIC por parte de profesores de Lenguaje, Matemática y Ciencias en educación media: Un estudio exploratorio. Proceedings from Segundo Congreso Interdisciplinatrio de Investigación en Educación, Santiago de Chile.

Larose, François, Grenon, Vincent, Morin, Marie Pier y Hasni, Abdelkrim. (2009). The impact of pre-service field training sessions on the probability of future teachers using ICT in school. European Journal of Teacher Education, 32(3), 289-303. doi:10.1080/02619760903006144

Lee, Chia-Jung y Kim, ChanMin. (2014). An implementation study of a TPACK-based instructional design model in a technology integration course. Education Tech Research Dev, 62(4), 437-460. doi:10.1007/s11423-014-9335-8

Lei, Jing. (2009). Digital Natives as Preservice Teachers: What Technology Preparation Is Needed? Journal of Computing in Teacher Education, 25(3), 87-97. Recuperado de http://www.eric.ed.gov/ERICWebPortal/detail?accno=EJ835233

Maio, Gregory y Haddock, Geoffrey. (2004). Theories of attitude. En Geoffrey Haddock and Gregory R. Maio (eds.), Contemporary perspectives on the psychology of attitudes (pp. 424-446). Hove, East Sussex; New York: Psychology Press. 
Marchesi, Álvaro y Martín, Elena. (2003). Tecnología y Aprendizaje. Investigación sobre el impacto del ordenador en el aula. Madrid: SM.

Margaryan, Anoush, Littlejohn, Allison y Vojt, Gabrielle. (2011). Are digital natives a myth or reality? University students' use of digital technologies. Computers \& Education, 56(2), 429-440. doi:10.1016/j.compedu.2010.09.004

Markauskaite, Lina. (2007). Exploring the structure of trainee teachers' ICT literacy: the main components of, and relationships between, general cognitive and technical capabilities. Educational Technology Research and Development, 55(6), 547-572. doi:10.1007/s11423-007-9043-8

Mayer, Richard. (2003). Learning and Instruction. Upper Saddle River, N.J: Merrill.

McDougall, A. (2008). Models and practices in teacher education programs for teaching with and about IT. En Joke Voogt and Gerald Knezek (Eds.), International Handbook of Information Technology in Primary and Secondary Education (Vol. 1, pp. 461-474). Estados Unidos de América: Springer US.

MENESR (Ministère de l'Éducation nationale, de l'Enseignement supérieur et de la Recherche) (2015). Compétences numériques. Qu'est-ce que les compétences numériques? Recuperado de https://c2i.enseignementsuprecherche.gouv.fr/enseignant/les-competences-numeriques

MINEDUC y ENLACES (Ministerio de Educación de Chile). (2011). Competencias y estándares TIC para la profesión docente. Santiago: MINEDUC. Recuperado de http://www.enlaces.cl/marco-de-competencias-tecnologicas-para-el-sistema-escolar/

Mumtaz, Shazia. (2000). Factors affecting teachers' use of information and communications technology: a review of the literature. Journal of Information Techology for Teacher Education, 9(3), 319-342. doi:10.1080/14759390000200096

$\mathrm{Ng}$, Wan. (2012). Can we teach digital natives digital literacy? Computers \& Education, 59(3), 1065-1078. doi:10.1016/j.compedu.2012.04.016

Niess, Margaret L. (2005). Preparing teachers to teach science and mathematics with technology: Developing a technology pedagogical content knowledge. Teaching and Teacher Education, 21(5), 509-523. Recuperado de https://www.sciencedirect.com/science/article/pii/S0742051X05000387

Niess, Margaret L. (2008). Guiding preservice teachers in Developing TPCK. En E. American Association of Colleges for Teacher (pp. 223-250). New York: Routledge for the American Association of Colleges for Teacher Education.

Oteiza Morra, Fidel. (2007). ¿Tienen impacto las tecnologías de información en los aprendizajes? Revista Enlaces, (1), 3-6. Recuperado de https://www.oei.es/historico/noticias/spip.php?article726 
Ottenbreit-Leftwich, Anne T., Glazewski, Krista D., Newby, Timothy J., y Ertmer, Peggy A. (2010). Teacher value beliefs associated with using technology: Addressing professional and student needs. Computers \& Education, 55(3), 1321-1335. doi:10.1016/j.compedu.2010.06.002

Petko, Dominik. (2012). Teachers' pedagogical beliefs and their use of digital media in classrooms: Sharpening the focus of the 'will, skill, tool' model and integrating teachers' constructivist orientations. Computers \& Education, 58(4), 1351-1359. doi:10.1016/j.compedu.2011.12.013

Prensky, Mark. (2011). Digital Wisdom and Homo Sapiens Digital. En Michael Thomas (ed.), Deconstructing digital natives : young people, technology, and the new literacies (Vol. 2, pp. 15-29). New York; London: Routledge.

Pšunder, Mateja, y Virtič, Mateja Ploj. (2010). The influence of the field of study on the use of modern information and communication technologies among student and teachers. Informatologia, 43(4), 269-279. Recuperado de http://hrcak.srce.hr/file/95941

Raman, Kumutha y Yamat, Hamidah. (2014). Barriers Teachers Face in Integrating ICT during English Lessons: A Case Study. Malaysian Online Journal of Educational Technology, 2(3), 11-19. Recuperado de https://files.eric.ed.gov/fulltext/EJ1086402.pdf

Rizza, Caroline. (2011). ICT and Initial Teacher Education: National Policies. OECD Education Working Papers, 61. doi: http://dx.doi.org/10.1787/5kg57kjj5hs8-en

Román, Marcela. (2010). Cuatro formas de incorporar las TIC a la enseñanza en el aula. En A. Bilbao y A. Salinas (Eds), El libro aboerto de la informática educativa: lecciones y desafío de la Red Enlaces (pp. 105-122). Santiago de Chile: ENLACES.

Rozo Sandoval, Ana Claudia, y Prada Dussán, Maximiliano. (2012). Panorama de la formación inicial docente y TIC en la Región Andina. Revista educación y pedagogía, 24(62), 191-204. Recuperado de http://www.iatreia.udea.edu.co/index.php/revistaeyp/article/viewFile/14203/12546

Salleh, Sallimah. (2016). Examining the influence of teachers ' beliefs towards technology integration in classroom. International Journal of Information and Learning Technology, 33(1), 17-35. doi:10.1108/IJILT-10-2015-0032

Sang, Guoyuan, Valcke, Martín, van Braak, Johan, y Tondeur, Jo. (2010). Student teachers' thinking processes and ICT integration: Predictors of prospective teaching behaviors with educational technology. Computers \& Education, 54(1), 103-112. doi:10.1016/j.compedu.2009.07.010

She, Hsiao-Ching y Liao, Ya-Wen. (2010). Bridging scientific reasoning and conceptual change through adaptative web-based learning. Journal of Research in Science Teaching, 47(1), 91-119. https://doi.org/10.1002/tea.20309

Siemens, George y Tittenberger, Peter. (2009). Handbook of emerging technologies for learning. Canada: University of Manitoba. 
Silva, Juan, Gros, Begoña, Garrido, José Miguel y Rodríguez, Jaime. (2006). Estándares en tecnologías de la información y la comunicación para la formación inicial docente: situación actual y el caso chileno. Revista Iberoamericana de Educación, 38(3),16-16.

Tapia Silva, Hugo. (2017). Orientaciones para la aplicación del Modelo TPACK en la formación de profesores de ERE. Revista Electrónica de Educación Religiosa, 7(1), 124.

Teo, Timothy. (2008). Pre-service teachers' attitude towards computer use: A Singapore survey. Australasian Journal of Educational Technology, 24(4), 413-424.

Teo, Timothy, Kabakçı Yurdakul, Işıl, y Ursavaş, Ömer Faruk. (2014). Exploring the digital natives among pre-service teachers in Turkey: a cross-cultural validation of the Digital Native Assessment Scale. Interactive Learning Environments, 24(6), 1231-1244. doi:10.1080/10494820.2014.980275

Thomas, Michael. (2011). Deconstructing digital natives: young people, technology, and the new literacies. New York; London: Routledge.

Tondeur, Jo, Hermans, Ruben, van Braak, Johan y Valcke, Martín. (2008). Exploring the link between teachers' educational belief profiles and different types of computer use in the classroom. Computers in Human Behavior, 24(6), 2541-2553. doi:10.1016/j.chb.2008.02.020

Unesco. (2011). UNESCO ICT Competency Framework for Teachers - Version 2.0. Paris: UNESCO.

Unesco. (2014). Enfoques estratégicos sobre las TIC en educación en América Latina y el Caribe. Santiago, Chile: OREALC/UNESCO Santiago de Chile. Recuperado de http://unesdoc.unesco.org/images/0022/002232/223251s.pdf

Webb, Mary y Cox, Margaret. (2004). A review of pedagogy related to information and communications technology. Technology, Pedagogy and Education, 13(3), 235-286. doi:10.1080/14759390400200183 